J. Appl. Numer. Optim. 1 (2019), No. 2, pp. 167-182

Available online at http://jano.biemdas.com

https://doi.org/10.23952/jano.1.2019.2.07

\title{
BEST APPROXIMATION, OPTIMAL RECOVERY, AND LANDAU INEQUALITIES FOR DERIVATIVES OF HUKUHARA-TYPE IN FUNCTION $L$-SPACES
}

\author{
VLADYSLAV BABENKO $^{1}$, VIRA BABENKO ${ }^{2, *}$ \\ ${ }^{1}$ Department of Mathematics, Dnipro National University, Ukraine \\ ${ }^{2}$ Department of Mathematics and Computer Science, Drake University, USA
}

\begin{abstract}
We consider the problem of approximation of unbounded positively homogeneous operators in $L$-spaces using Lipschitz operators. We study its connection to the problem of computing modulus of continuity of the unbounded operator on the class of elements, as well as, to the problem of optimal recovery of an unbounded operator by a Lipschitz one on the class of elements given with an error. Moreover, in $L$-spaces and for positively homogeneous operators, the connection of the above-mentioned problems with inequalities of Landau - Kolmogorov type is studied. As applications, we consider the problem of approximation of unbounded operator, that for functions with values in some $L$-space puts in a correspondence Hukuhara-type derivatives, by Lipschitz operators. In addition, we compute the modulus of continuity of this operator and obtain exact Landau-Kolmogorov type inequalities. Further, we solve the problem of the optimal recovery of this operator on the class of functions that have Hukuhara-type derivative with the given majorant of the modulus of continuity (in the case of optimal recovery, elements of this class are given with an error).
\end{abstract}

Keywords. $L$-spaces; Derivatives of Hukuhara-type; Best approximation; Optimal recovery; Lipschitz operators.

2010 Mathematics Subject Classification. 26D10; 28B20; 41A44.

\section{INTRODUCTION}

Many problems of the approximation theory and numerical analysis are reduced to the study of the deviation of the operator $A$ (bounded or not) from a fixed (usually bounded) operator $T$ on a given class of elements of the normed space. In particular, these are the problems of obtaining error estimates for various numerical differentiation formulas. Wherein, the problem of choosing the optimal (in some sense) approximating operator arises naturally.

The problem of the best approximation of an unbounded operator by a bounded operator on the given class of elements was formulated by Stechkin [13] (see also [1]). He also obtained first results on the solution of this problem. This problem is closely related to the problem on obtaining inequalities for the norms of consecutive derivatives (norms of powers of an unbounded operator, or, more generally, the problem of comparing operators) and with ill-posed problem of recovery of the values of unbounded operator on the class of elements known with some error (see $[1,3]$ on the connection). An example of such a problem is the problem of obtaining stable formulas for numerical differentiation (see [11, 12] and references therein).

\footnotetext{
${ }^{*}$ Corresponding author.
}

E-mail addresses: babenko.vladislav@gmail.com (V. Babenko), vira.babenko@drake.edu (Vira Babenko)

Received July 16, 2019; Accepted August 13, 2019.

(C)2019 Journal of Applied and Numerical Optimization 
The purpose of this work is to consider these problems in the case of positively homogeneous operators in $L$-spaces. The notion of an $L$-space was introduced by Vahrameev [14]. He also defined integration for functions with values in $L$-spaces. The use of this notion let us consider many questions connected to set-valued and fuzzy-valued functions from a common point of view.

This paper is organized in the following way. In Section 2, we present the definition of $L$-spaces and, for completeness, give some examples.

We define a notion of boundedness and unboundedness for positively homogeneous operators in $L$ spaces. Besides that, we define Lipschitz operators (any Lipschitz operator is bounded). In Section 3, we study analog of Stechkin's problem [13] for the best approximation of unbounded positively homogeneous operators in $L$-spaces by Lipschitz operators. In addition, we consider its connection to the problem of computing modulus of continuity of the operator on the class of elements, as well as, the problem of optimal recovery of an unbounded operator on the class of elements given with an error. Moreover, in $L$ - spaces for positively homogeneous operators, a connection with inequalities of Landau-Kolmogorov type is studied.

In Section 4, we present the necessary definitions and facts for analysis of functions with values in $L$-spaces. In particular, we define the notion of Hukuhara-type difference for elements of $L$-space (Hukuhara [9] defined this notion in the space of sets) and we define Hukuhara-type derivatives for functions with values in $L$-spaces. Furthermore, we provide a definition of integrals of such functions as well as all necessary properties of introduced notions. Note that in the paper [5] the calculus of functions with values in $L$-spaces was built and existence and uniqueness of solutions of integral (and differential) equations for such functions were studied. Hukuhara derivative is important for a number of applications. For instance, differential equations and differential inclusions with Hukuhara derivatives are studied by many authors. Similar questions are of interest for derivatives of the Hukuhara-type. One of the examples of applications of derivatives of the Hukuhara-type is as follows. In order to solve an integral equation

$$
f(t)=\int_{0}^{t} x(u) d u
$$

(here $f$ is known, and $x$ is an unknown function with values in $L$-space) it becomes necessary to calculate the derivative of the Hukuhara-type from the function $f$. This naturally raises questions about the formulas for the approximate calculation of a derivative of the Hukuhara-type and their stability.

In Sections 5 and 6, we are solving the problem of approximation of unbounded operator, that for the function $x$ defined on $\mathbb{R}_{+}$or $\mathbb{R}$ with values in $L$-spaces, puts in a correspondence a Hukuhara-type derivative, by Lipschitz operators. We also compute the modulus of continuity of this operator and obtain exact Landau-Kolmogorov-type inequalities. In addition, we solve the problem of the optimal recovery of this operator on the class of functions that have Hukuhara-type derivative with the given majorant of the modulus of continuity (in the case of optimal recovery, elements of this class are given with an error).

\section{2. $L$-SPACES}

2.1. Definitions. The following definition was introduced by Vahrameev in [14]:

Definition 2.1. A complete separable metric space $X$ with metric $d=d_{X}$ is said to be an $L$ - space if in $X$ operations of addition of elements and their multiplication with real numbers are defined, and the following axioms are satisfied: 
Axiom 1. $\forall x, y \in X \quad x+y=y+x$;

Axiom 2. $\forall x, y, z \in X \quad x+(y+z)=(x+y)+z$;

Axiom 3. $\exists \theta \in X \forall x \in X \quad x+\theta=x$ (where $\theta$ is called a zero element in $X$ );

Axiom 4. $\forall x, y \in X \quad \lambda \in \mathbb{R} \quad \lambda(x+y)=\lambda x+\lambda y$;

Axiom 5. $\forall x \in X \quad \lambda, \mu \in \mathbb{R} \quad \lambda(\mu x)=(\lambda \mu) x$

Axiom 6. $\forall x \in X \quad 1 \cdot x=x, \quad 0 \cdot x=\theta$

Axiom 7. $\forall x, y \in X \quad \lambda \in \mathbb{R} \quad d(\lambda x, \lambda y)=|\lambda| d(x, y)$;

Axiom 8. $\forall x, y, u, v \in X \quad d(x+y, u+v) \leq d(x, u)+d(y, v)$.

Definition 2.2. We call an $L$-space $X$ isotropic if

$$
\forall x, y, u \in X \quad d(x+u, y+u)=d(x, y) .
$$

Below we consider isotropic $L$ spaces only.

\subsection{Examples of isotropic $L$-spaces.}

(1) Any real and separable Banach space $\left(Y,\|\cdot\|_{Y}\right)$ endowed with the metric $d(x, y)=\|x-y\|_{Y}$.

(2) Let $\mathscr{K}\left(\mathbb{R}^{n}\right)$ be the set of all nonempty and compact subsets of $\mathbb{R}^{n}$ and let $\mathscr{K}^{c}\left(\mathbb{R}^{n}\right) \subset \mathscr{K}\left(\mathbb{R}^{n}\right)$ be the subset of convex sets.

Definition 2.3. Let $|\cdot|$ be the Euclidean norm in $\mathbb{R}^{n}$. For $A, B \in \mathscr{K}\left(\mathbb{R}^{n}\right)$, and $\alpha \in \mathbb{R}$ :

$$
A+B:=\{x+y: x \in A, y \in B\} ; \alpha A:=\{\alpha x: x \in A\}
$$

and Hausdorff Distance

$$
d_{\mathscr{K}\left(\mathbb{R}^{n}\right)}(A, B)=\max \left\{\sup _{x \in A} \inf _{y \in B}|x-y|, \sup _{x \in B} \inf _{y \in A}|x-y|\right\} .
$$

With these operations and metric, $\mathscr{K}\left(\mathbb{R}^{n}\right)$ and its subspace $\mathscr{K}^{c}\left(\mathbb{R}^{n}\right)$ are complete, separable metric spaces (see [8]) and since the axioms 1-8 as well as condition of Definition 2.2 hold, these spaces are isotropic $L$-spaces.

(3) Any complete and separable quasilinear normed space $Y$ (see the definition in [2]) is an $L$-space.

(4) Consider (see, e.g., [7]) the class of fuzzy sets $\mathscr{E}^{n}$ consisting of functions $u: \mathbb{R}^{n} \rightarrow[0,1]$ such that

(a) $u$ is normal, i.e., there exists an $x_{0} \in \mathbb{R}^{n}$ such that $u\left(x_{0}\right)=1$;

(b) $u$ is fuzzy convex, i.e., for any $x, y \in \mathbb{R}^{n}$ and $0 \leq \lambda \leq 1$,

$$
u(\lambda x+(1-\lambda) y) \geq \min \{u(x), u(y)\}
$$

(c) $u$ is upper semicontinuous;

(d) the closure of $\left\{x \in \mathbb{R}^{n}: u(x)>0\right\}$, denoted by $[u]^{0}$, is compact.

For each $0<\alpha \leq 1$, the $\alpha$-level set $[u]^{\alpha}$ of a fuzzy set $u$ is defined as $[u]^{\alpha}=\left\{x \in \mathbb{R}^{n}: u(x) \geq \alpha\right\}$.

The addition $u+v$ and scalar multiplication $c u, c \in \mathbb{R} \backslash\{0\}$, on $\mathscr{E}^{n}$ are defined, in terms of $\alpha$-level sets, by

$$
[u+v]^{\alpha}=[u]^{\alpha}+[v]^{\alpha}, \quad[c u]^{\alpha}=c[u]^{\alpha} \text { for each } 0<\alpha \leq 1 .
$$


Define also $0 \cdot u$ by the equality $[0 \cdot u]^{\alpha}=\{\theta\}$ (here $\theta=(0, \ldots, 0) \in \mathbb{R}^{n}$ ). One of the possible metrics in $\mathscr{E}^{n}$ is defined in the following way

$$
d_{\mathscr{E}^{n}, p}(u, v)=\left(\int_{0}^{1} d_{\mathscr{K}\left(\mathbb{R}^{n}\right)}\left([u]^{\alpha},[v]^{\alpha}\right)^{p} d \alpha\right)^{1 / p}, \quad 1 \leq p<\infty .
$$

Then the space $\left(\mathscr{E}^{n}, d_{\mathscr{E}^{n}, p}\right)$ is (see [7, Theorem 3]) a complete separable metric space and therefore an $L$-space.

(5) Spaces of continuous mappings. The structure of $L$-spaces arises naturally in some spaces of mappings with values in $L$-spaces. For example, let a compact topological space $G$ and $L$-space $X$ be given. Denote by $C(G, X)$ the space of all continuous mappings $f: G \rightarrow X$. Operations of addition and multiplication by real numbers are defined in $C(G, X)$ in a standard way. Metric in $C(G, X)$ is defined by the relation

$$
d_{C(G, X)}\left(f_{1}, f_{2}\right):=\max _{t \in T} d_{X}\left(f_{1}(t), f_{2}(t)\right) .
$$

It is clear that $\left(C(G, X), d_{C(G, X)}\right)$ is an $L$-space. We will also consider spaces $C(G, X)$ of continuous bounded functions $f: G \rightarrow X$ with noncompact $G$. In particular, we will consider spaces $C(\mathbb{R}, X)$ and $C\left(\mathbb{R}_{+}, X\right)$. Such spaces are complete, but not separable.

2.3. Convex and invertible elements of $L$ - spaces. We need the following notions of convex elements in $L$-spaces.

Definition 2.4. An element $x \in X$ is convex if $\lambda x+\mu x=(\lambda+\mu) x, \forall \lambda, \mu \geq 0$.

Remark 2.5. Note that if the element $x$ is convex, then it follows from axioms 7 and 8 that $\forall \lambda, \mu \in \mathbb{R}$

$$
d_{X}(\lambda x, \mu x) \leq|\lambda-\mu| d_{X}(x, \theta) .
$$

Let $X^{c}$ be a set of all convex elements of a given $L$-space $X$.

Remark 2.6. $X^{c}$ is a closed subset of $X$.

Definition 2.7. An element $x \in X$ is invertible if there exist an element $x^{\prime} \in X$ such that $x+x^{\prime}=\theta$ ( $x^{\prime}$ is called an inverse to $x$ ). The set of all invertible elements of the space $X$ we denote by $X^{\text {inv }}$.

In what follows, we assume that $X^{\text {inv }} \cap X^{\mathrm{c}} \neq\{\theta\}$.

We need the following Lemmas.

Lemma 2.8. If $x \in X^{\text {inv }}$, then inverse element $x^{\prime}$ is unique.

Lemma 2.9. If $x \in X^{\text {inv }} \cap X^{\mathrm{c}} \neq \emptyset$, then $x^{\prime} \in X^{\mathrm{c}}$ and, moreover,

$$
\forall \alpha, \beta \in \mathbb{R} \quad(\alpha+\beta) x^{\prime}=\alpha x^{\prime}+\beta x^{\prime}
$$

\section{STEChKin's ANd Related PRoblems in $L$ - SPACES}

We use the following notation:

$$
\|x\|_{X}=d_{X}(x, \theta) .
$$

Definition 3.1. Nonempty subset $K$ of an $L$-space $X$ is called a cone, if $\forall x \in$ and $\forall \alpha \geq 0, \alpha x \in K$. 
Definition 3.2. Let $L$-spaces $X, Y$ be given and let $K \subset X$ be cone in $X$. Let also positively homogeneous operator $T: K \rightarrow Y$ be given. We say that this operator is bounded on $K$ if

$$
\exists M>0, \forall x \in K \quad\|T x\|_{Y} \leq M\|x\|_{X}
$$

In the opposite case, we say that operator $T$ is unbounded. We denote by $\mathscr{L}(K, Y)$ a set of all positively homogeneous, bounded operators $T: K \rightarrow Y$. The smallest value $M$ for which the above inequality holds for any $x \in K$, similarly to the case of normed spaces, we call the norm of operator $A$ and denote by $\|A\|_{\mathscr{L}}$.

Definition 3.3. An operator $T: K \rightarrow Y$ is a Lipschitz operator if

$$
\exists M>0 \forall x^{\prime}, x^{\prime \prime} \in X \quad d_{Y}\left(T\left(x^{\prime}\right), T\left(x^{\prime \prime}\right) \leq M d_{X}\left(x^{\prime}, x^{\prime \prime}\right) .\right.
$$

We denote by $\mathscr{L} i p(K, Y)$ the set of all Lipschitz operators $T: K \rightarrow Y$. The smallest constant $M$, for which (3.1) holds, is called the Lipschitz norm of mapping $f$ and we denote it by $\|T\|_{\mathscr{L} \text { ip }}$.

Note that $\mathscr{L} i p(K, Y) \subset \mathscr{L}(K, Y)$ and for any $T \in \mathscr{L} i p(K, Y)$,

$$
\|T\|_{\mathscr{L}} \leq\|T\|_{\mathscr{L} i p}
$$

Indeed,

$$
\|T x\|_{Y}=d_{Y}(T x, \theta)=d_{Y}(T x, T \theta) \leq\|T\|_{\mathscr{L} i p} d_{X}(x, \theta)=\|T\|_{\mathscr{L} i p}\|x\|_{X} .
$$

Let some class of elements $Q \subset K$, such that $\theta \in Q$, be given. Note that class $Q$ can usually be defined as follows. Let positively homogeneous functional $F: K \rightarrow \mathbb{R}_{+}$, such that $\operatorname{Ker}(F) \subset \operatorname{Ker}(A)$ be given. Then

$$
Q=Q_{F}=\{x \in K: F(x) \leq 1\} .
$$

We formulate generalized Stechkin's problem on approximation of unbounded operators on the given class $Q$ of elements from $K$. As a set of approximating operators, we consider the set of Lipschitz operators. Therefore, we use the following notations below:

$$
\mathscr{L}=\mathscr{L} i p \text { and }\|T\|=\|T\|_{\mathscr{L} i p}
$$

Let $L$-spaces $X, Y$, as well as, positively homogeneous, unbounded operator $A: K \rightarrow Y$ and a class of elements $Q \subset K$ be given.

Definition 3.4. For bounded operator $T: K \rightarrow Y$ the value

$$
U(T)=U(A, T, Q)=\sup _{x \in Q} d_{Y}(A x, T x) .
$$

is called deviation of operator $T$ from operator $A$ on $Q$.

Definition 3.5. For $N>0$,

$$
E(N)=E_{\mathscr{L}}(A, N, Q)=\inf _{\|T\| \leq N} U(T) .
$$

The value $E(N)$ is called the best approximation of operator $A$ by operators $T \in \mathscr{L}$ such that $\|T\| \leq N$. 
Generalized Stechkin's Problem consists of finding the value $E(N)$ and operator, that realizes inf in the right side of the (3.2).

If values $U(T)$ or $E(N)$ are known, then we obtain the following abstract versions of Landau - Kolmogorov inequalities in additive form:

$$
\forall x \in Q \quad\|A x\|_{Y} \leq U(T)+\|T\|\|x\|_{X}
$$

and

$$
\forall x \in Q \quad\|A x\|_{Y} \leq E(N)+N\|x\|_{X},
$$

that in the case $Q=Q_{F}$ leads to inequalities

$$
\forall x \in \operatorname{cone}(Q) \quad\|A x\|_{Y} \leq U(T) F(x)+\|T\|\|x\|_{X}
$$

and

$$
\forall x \in \operatorname{cone}(Q) \quad\|A x\|_{Y} \leq E(N) F(x)+N\|x\|_{X}
$$

(here cone $(Q)=\left\{\alpha x: x \in Q, \alpha \in \mathbb{R}_{+}\right\}$).

Definition 3.6. Let

$$
\Omega(\delta)=\Omega(\delta, A, Q):=\sup _{x \in Q,\|x\|_{X} \leq \delta}\|A x\|_{Y}, \delta \geq 0 .
$$

Such function is called modulus of continuity of operator $A$ on the class of elements $Q \subset K$.

From inequality (3.3), we immediately obtain the following estimation for the modulus of continuity of operator $A$ on the class of elements $Q \subset K$ :

$$
\Omega(\delta) \leq \inf _{N>0}\{E(N)+N \delta\} .
$$

If function $\Omega(\delta)$ is known and $Q=Q_{F}$, then, for any $x \in K, x \notin \operatorname{Ker}(F), x / F(x) \in Q_{F}$, and

$$
\frac{\|A x\|_{Y}}{F(x)} \leq \Omega\left(\frac{\|x\|_{X}}{F(x)}\right) .
$$

Therefore,

$$
\|A x\|_{Y}=\Omega\left(\frac{\|x\|_{X}}{F(x)}\right) F(x) .
$$

This is an abstract version of Landau-Kolmogorov-type inequalities for intermediate derivatives in multiplicative form. If $\Omega(\delta)=M \cdot \delta^{\alpha}$, then

$$
\|A x\|_{Y}=M\|x\|_{X}^{\alpha} F(x)^{1-\alpha} .
$$

For any operator $T,\|T\| \leq N$, and any $x \in Q$, we have

$$
d_{Y}(A x, T x) \geq\|A x\|_{y}-\|T x\|_{Y} \geq\|A x\|_{Y}-\|T\|\|x\|_{X} .
$$

Consequently, for any $\delta \geq 0$, we have

$$
U(T) \geq \sup _{x \in Q,\|x\|_{X} \leq \delta}\|A x\|_{Y}-N \delta=\Omega(\delta)-N \delta .
$$

We obtain a useful lower bound for $E(N)$ :

$$
E(N) \geq \sup _{\delta \geq 0}(\Omega(\delta)-N \delta)
$$


Many problems of computational mathematics, the theory of functions and other branches of mathematics are ill-posed problems of recovering of values of an unbounded operator $A$ on elements of the class $Q \subset D(A)$ in assumption that elements of the class $Q$ are given with known error. We will solve this problem using operators $T \in \mathscr{L}$ for recovery.

Let us formulate the problem of optimal recovery of operator $A$ on the class $Q$.

For number $\delta \geq 0$ and operator $T \in \mathscr{L}$, we set

$$
U_{\delta}(T)=U_{\delta}(T, A, Q)=\sup \left\{d_{Y}(A x, T \eta): x \in Q, \eta \in X, d_{X}(x, \eta) \leq \delta\right\} .
$$

Then,

$$
\mathscr{E}_{\delta}(\mathscr{L})=\mathscr{E}_{\delta}(\mathscr{L}, A, Q)=\inf _{T \in \mathscr{L}} U_{\delta}(T)
$$

is the value of the best recovery of operator $A$ using the methods of recovery $\mathscr{L}$ on elements of the class $Q$ given with an error $\delta$. The task is to find this quantity and operator $T$ that realizes inf in the right-hand side.

If for some operator $T \in \mathscr{L}$ we know value of $U(T)$, then for $U_{\delta}(T)$ we get an estimate

$$
U_{\delta}(T) \leq U(T)+\|T\| \delta .
$$

Indeed,

$$
d_{Y}(A x, T \eta) \leq d_{Y}(A x, T x)+d_{Y}(T x, T \eta) \leq U(T)+\|T\| d_{X}(x, \eta) \leq U(T)+\|T\| \delta .
$$

Therefore, for any $N>0$,

$$
\mathscr{E}_{\delta}(\mathscr{L}) \leq E(N)+N \delta
$$

Finally,

$$
\mathscr{E}_{\delta}(\mathscr{L}) \leq \inf _{N>0}(E(N)+N \delta)
$$

Next, if $Q$ contains $\theta$, then, for any $T \in \mathscr{L}(X, Y)$,

$$
\begin{aligned}
U_{\delta}(T) & =\sup _{x \in Q} \sup _{d_{X}(x, \eta) \leq \delta} d_{Y}(A x, T \eta) \\
& \geq \sup _{x \in Q,\|x\|_{X} \leq \delta} d_{Y}(A x, T \theta) \\
& =\sup _{x \in Q,\|x\|_{X} \leq \delta}\|A x\|_{Y}=\Omega(\delta) .
\end{aligned}
$$

Hence,

$$
\mathscr{E}_{\delta}(\mathscr{L}) \geq \Omega(\delta)
$$

Summing up the above, we see that the following theorem that establishes connections between the Stechkin problem, Landau-Kolmogorov inequalities in additive form, the problem of calculating the modulus of continuity of operator, and the problem of optimal recovery of the operator on a class of elements defined with a known error is valid.

Theorem 3.1. Let $A: K \rightarrow Y$ be a positively homogeneous operator and $Q \subset K$. Then, for any Lipschitz operator $T$,

$$
\|A x\|_{Y} \leq U(T)+\|T\| \cdot\|x\|_{X}
$$

and, for any $N$,

$$
\|A x\|_{Y} \leq E(N)+N \delta
$$


For any $\delta$,

$$
\begin{gathered}
\Omega(\delta) \leq \inf (E(N)+N \delta), \\
E(N) \geq \sup _{\delta \geq 0}(\Omega(\delta)-N \delta), \\
\Omega(\delta) \leq \mathscr{E}_{\delta}(\mathscr{L}) \leq \inf _{N>0}(E(N)+N \delta) .
\end{gathered}
$$

The following theorem complements the previous one.

Theorem 3.2. If there exists an element $\bar{x}$ and an operator $\bar{T}$ such that

$$
\|A \bar{x}\|_{Y}=U(\bar{T})+\|\bar{T}\| \cdot\|\bar{x}\|_{X}
$$

then

$$
\begin{gathered}
E(\|\bar{T}\|)=U(\bar{T}), \\
\Omega\left(\|\bar{x}\|_{X}\right)=\|A \bar{x}\|_{Y}=\mathscr{E}_{\|\bar{x}\|_{X}}(\mathscr{L}) .
\end{gathered}
$$

Let us prove Theorem 3.2. Using (3.5) and (3.7), we have

$$
E(\|\bar{T}\|) \geq\|A \bar{x}\|-\|\bar{T}\|\|\bar{x}\|=U(\bar{T}) .
$$

Since the opposite inequality is obvious, equality (3.8) is proved.

Further,

$$
\Omega\left(\|\bar{x}\|_{X}\right) \leq \inf _{N>0}\left(E(N)+N\|\bar{x}\|_{X}\right) \leq E(\|\bar{T}\|)+\|\bar{T}\|\|\bar{x}\|_{X}=U(\bar{T})+\|\bar{T}\|\|\bar{x}\|_{X}=\|A \bar{x}\| \leq \Omega\left(\|\bar{x}\|_{X}\right) .
$$

We have proved that

$$
\Omega\left(\|\bar{x}\|_{X}\right)=\|A \bar{x}\|
$$

Finally, using (3.6), we obtain

$$
\Omega(\|\bar{x}\|) \leq \mathscr{E}_{\|\bar{x}\|_{X}}(\mathscr{L}) \leq E(\|\bar{T}\|)+\|\bar{T}\|\|\bar{x}\|_{X}=U(\bar{T})+\|\bar{T}\|\|\bar{x}\|_{X}=\|A \bar{x}\| \leq \Omega(\|\bar{x}\|) .
$$

Theorem is proved.

\section{Elements of CALCUluS IN $L$-SPACES}

In this section, we present without proof some statements most of which were obtained in the paper [5].

4.1. Hukuhara-type difference and its properties. The notion of the Hukuhara difference of two sets was introduced by Hukuhara in [9]. Here we generalize it as the Hukuhara-type difference for elements in $L$-spaces. Everywhere below we suppose that $X$ consists only of convex elements.

Definition 4.1. We say that an element $z \in X$ is the Hukuhara-type difference of elements $x, y \in X$, if $x=y+z$. We denote this difference by $z=x-\frac{h}{-} y$.

The following properties of Hukuhara difference of sets are known (see, e.g., [6, 10]). Similar properties for a general Hukuhara-type difference in $L$-spaces were proved in [5].

Lemma 4.2. Let $x, y, u, v \in X$. The Hukuhara-type difference has the following properties

(1) $\forall x, y$ difference $(x+y) \stackrel{h}{-} x$ exist and $(x+y) \stackrel{h}{-} x=y$.

(2) If for $x$ and $y$ the Hukuhara-type difference $x-\frac{h}{-} y$ exist, then it is unique. 
(3) If $u \stackrel{h}{-} v$ and $(x+u) \stackrel{h}{-} v$ exist, then $x+(u \stackrel{h}{-v})=(x+u) \stackrel{h}{-} v$.

(4) If $x \stackrel{h}{-} v$ and $v \stackrel{h}{-} u$ exist, then $x \stackrel{h}{-} u$ exists and $x \stackrel{h}{-} u=(x \stackrel{h}{-} v)+(v-u)$

(5) If $x \stackrel{h}{-} y$ exists, then for any number $\alpha$ we have $\alpha x \stackrel{h}{-} \alpha y=\alpha\left(x-\frac{h}{-} y\right)$.

(6) If $x \stackrel{h}{-} u$ and $y \stackrel{h}{-} v$ exist, then $(x+y) \stackrel{h}{-}(u+v)$ exists and the following equality holds $(x+y) \stackrel{h}{-}(u+v)=(x-u)+\left(y-\frac{h}{-} v\right)$.

(7) If $x \stackrel{h}{-} y, v \stackrel{h}{-} u$, and $y \stackrel{h}{-} v$ exist, then $x \stackrel{h}{-} u$ and $(x-u) \stackrel{h}{-}(y \stackrel{h}{-} v)$ exist and $(x-u) \stackrel{h}{-}(y-v)=(x-y)+(v-u)$.

(8) If $x \stackrel{h}{-} y, u \stackrel{h}{-} v, y \stackrel{h}{-} v$ and $(x \stackrel{h}{-} y) \stackrel{h}{-}(u \stackrel{h}{-} v)$ exist, then $x \stackrel{h}{-} u$ and $(x \stackrel{h}{-} u) \stackrel{h}{-}(y \stackrel{h}{-} v)$ exist, and $(x-u) \stackrel{h}{-}\left(y-\frac{h}{-v}\right)=(x-y) \stackrel{h}{-}(u-\underline{h})$.

(9) If $x-y$ exists, then $d_{X}(x, y) \leq d_{X}(x-y, \theta)$. Moreover, if space $X$ is isotropic, then $d_{X}(x, y)=$ $d_{X}(x-y, \theta)$.

(10) If $x \stackrel{h}{-} u, y \stackrel{h}{-} v$ exist and space $X$ is isotropic, then $d_{X}(x \stackrel{h}{-} u, y \stackrel{h}{-} v) \leq d_{X}(x, y)+d_{X}(u, v)$.

(11) If $x \stackrel{h}{-} u$ exists then for any $\alpha \in(0,1)$ the difference $x-(\alpha u)$ exists and $x \stackrel{h}{-}(\alpha u)=\alpha\left(x-\frac{h}{-} u\right)+$ $(1-\alpha) x$.

4.2. Hukuhara-type derivative and its properties. The notion of the Hukuhara derivative of setvalued functions was introduced by Hukuhara in [9], and one can find properties of Hukuhara derivative for set-valued functions, for example, in [10].

Definition 4.3. If $t \in(a, b)$ and for all small enough $\gamma>0$ there exist differences $f(t+\gamma) \stackrel{h}{-} f(t)$ and $f(t) \stackrel{h}{-} f(t-\gamma)$, and both limits $\lim _{\gamma \rightarrow 0^{+}} \frac{f(t+\gamma)-f(t)}{\gamma}$ and $\lim _{\gamma \rightarrow 0^{+}} \frac{f(t)-f(t-\gamma)}{\gamma}$ exist and are equal to each other, then the function has a Hukuhara-type derivative $D_{H} f(t)$ at the point $t$ (if $t=a$ or $t=b$ then there exists only one limit) and $D_{H} f(t):=\lim _{\gamma \rightarrow 0^{+}} \frac{f(t+\gamma) \stackrel{h}{\gamma} f(t)}{\gamma}$.

Remark 4.4. If $f$ has Hukuhara-type derivative at point $t_{0}$, then $f$ is continuous at point $t_{0}$.

Lemma 4.5. (1) If functions $f, g:[a, b] \rightarrow X$ have Hukuhara-type derivatives at point $t \in[a, b]$, then the derivative of their sum exists and $D_{H}(f+g)(t)=D_{H} f(t)+D_{H} g(t)$.

(2) Let $f, g:[a, b] \rightarrow X^{c}$ be such that for any $t \in[a, b], D_{H} f(t), D_{H} g(t)$, and difference $f(t) \stackrel{h}{-} g(t)$ exist. Assume that, for any $t^{\prime}, t^{\prime \prime} \in[a, b]\left(t^{\prime}<t^{\prime \prime}\right)$, there exists

$$
\left(f\left(t^{\prime \prime}\right) \stackrel{h}{-} f\left(t^{\prime}\right)\right) \stackrel{h}{-}\left(g\left(t^{\prime \prime}\right) \stackrel{h}{-} g\left(t^{\prime}\right)\right) \text {. }
$$

Then there exist $D_{H}(f(t) \stackrel{h}{-} g(t))$ and $D_{H}(f(t) \stackrel{h}{-} g(t))=D_{H} f(t) \stackrel{h}{-} D_{H} g(t)$. 
(3) Let $f:[a, b] \rightarrow X$ have a Hukuhara-type derivative $D_{H} f$ on the interval $[a, b]$. Let also $\varphi:[c, d] \rightarrow$ $[a, b]$ be a strictly increasing real-valued function, differentiable at any point on $[c, d]$. Then $f \circ \varphi$ has a Hukuhara-type derivative $D_{H}$ at every point on $[c, d]$ and

$$
D_{H}(f \circ \varphi)(t)=D_{H} f(\varphi(t)) \varphi^{\prime}(t) .
$$

(4) Let a real-valued function $f(t)$ (differentiable, nonnegative, and nondecreasing) and a differentiable in Hukuhara sense function $F(t)$ with convex values in $L$-space be given. Then $f(t) F(t)$ has Hukuhara-type derivative and

$$
D_{H}(f F)(t)=f^{\prime}(t) F(t)+f(t) D_{H} F(t) .
$$

4.3. Integrals of functions with values in $L$-spaces and its properties. Riemannian integral for functions with values in $L$-space was defined by Vahrameev in [14] (see also [2]). Such integral was used by one of the authors of this work in [4] while studying methods of approximate solution of linear integral equations for functions with values in $L$-spaces. We need this integral for convex-valued functions. For such functions Riemannian integral can be defined in a standard way. So we omit this definition and present only several properties of the integral. Detailed proof of these properties in general case can be found in [5].

4.3.1. Elementary Properties. The Riemannian integral for functions with values in $L$-space $X$ has the following properties:

(1) If $f$ and $g$ are integrable, then for any $\alpha, \beta \in \mathbb{R}$ the function $\alpha f+\beta g$ is integrable, and moreover

$$
\int_{a}^{b}(\alpha f(t)+\beta g(t)) d t=\alpha \int_{a}^{b} f(t) d t+\beta \int_{a}^{b} g(t) d t
$$

(2) If $f$ and $g$ are integrable, then the function $t \rightarrow d_{X}(f(t), g(t))$ is integrable and

$$
d_{X}\left(\int_{a}^{b} f(t) d t, \int_{a}^{b} g(t) d t\right) \leq \int_{a}^{b} d_{X}(f(t), g(t)) d t .
$$

(3) If $f$ is integrable on $[a, b]$, and $a \leq c \leq b$, then function $f$ is integrable on $[a, c]$ and $[c, b]$ and

$$
\int_{a}^{b} f(t) d t=\int_{a}^{c} f(t) d t+\int_{c}^{b} f(t) d t
$$

(4) If functions $f$ and $g$ are integrable on $[a, b]$ and for any $t \in[a, b]$ the difference $f(t) \stackrel{h}{-} g(t)$ exists, then the difference $f(t) \stackrel{h}{-} g(t)$ is integrable on $[a, b]$ and

$$
\int_{a}^{b}(f(t) \stackrel{h}{-} g(t)) d t=\int_{a}^{b} f(t) d t-\int_{a}^{b} g(t) d t .
$$

4.3.2. Derivative of an integral with a variable upper limit and analog of fundamental Theorem of calculus.

Lemma 1. Let $X$ be an $L$-space with convex elements $f \in C([a, b], X)$. Then as $\gamma \rightarrow 0^{+}$

$$
\gamma^{-1}\left(\int_{a}^{t+\gamma} f(s) d s-\int_{a}^{t} f(s) d s\right) \text { and } \gamma^{-1}\left(\int_{a}^{t} f(s) d s-\int_{a}^{t-\gamma} f(s) d s\right)
$$

go to $f(t)$ uniformly with respect to $t$. Besides,

$$
D_{H}\left(\int_{a}^{x} f(t) d t\right)=f(x), x \in[a, b]
$$


Theorem 4.1. If function $F:[a, b] \rightarrow X$ has a continuous Hukuhara-type derivative $D_{H} F(t)$ on $[a, b]$ and conditions

$$
\gamma^{-1}(F(t+\gamma) \stackrel{h}{-} F(t)) \rightrightarrows D_{H} F(t) \text { and } \gamma^{-1}(F(t) \stackrel{h}{-} F(t-\gamma)) \rightrightarrows D_{H} F(t), \gamma \rightarrow 0^{+}
$$

(by $\rightrightarrows$ we denote a uniform convergence with respect to $t$ ) are satisfied, then $\forall t \in[a, b]$ the equality

$$
F(t)=F(a)+\int_{a}^{t} D_{H} F(s) d s, t \in[a, b]
$$

holds.

\section{Stechion's and Related Problems in the Space $C\left(\mathbb{R}_{+}, X\right)$}

Everywhere below $G=\mathbb{R}$ or $G=\mathbb{R}_{+}$. Let $X$ be some $L$-space with convex elements.

We use the following notations. For bounded function $x(\cdot) \in C(G, X)$, let

$$
\|x(\cdot)\|_{C(G, X)}:=\sup _{t \in G}\|x(t)\|_{X} .
$$

Let $\omega(t)$ be some modulus of continuity (i.e. non-decreasing on $\mathbb{R}_{+}$, continuous and semiadditive function such that $\omega(0)=0)$. Denote by $H^{\omega}(G, X)$ the set of functions $x \in C(G, X)$ such that

$$
\|x\|_{H^{\omega}(G, X)}:=\sup _{u, v \in G ; u \neq v} \frac{d_{X}(x(u), x(v))}{\omega(|u-v|)}<\infty .
$$

By $B H^{\omega}(G, X)$, we denote the set of functions $x \in H^{\omega}(G, X)$ such that $\|x\|_{H^{\omega}(G, X)} \leq 1$. Finally, by $W^{1} B H^{\omega}(G, X)$, we denote the set of functions $x \in C(G, X)$ such that $D_{H} x \in B H^{\omega}(G, X)$ and set

$$
W^{1} H^{\omega}(G, X)=\operatorname{cone}\left(W^{1} B H^{\omega}(G, X)\right) .
$$

With the notations used in Section 3, we have

$$
K=\left\{x \in C(G, X): D_{H} x \in C(G, X)\right\}
$$

and

$$
Q=W^{1} B H^{\omega}(G, X) .
$$

In this section, $G=\mathbb{R}_{+}$. For any $\gamma>0$, we define an operator $T_{\gamma}: K \rightarrow C\left(\mathbb{R}_{+}, X\right)$ :

$$
T_{\gamma} x(t)=\gamma^{-1}(x(t+\gamma) \stackrel{h}{-} x(t)) .
$$

We now estimate the norm of $T_{\gamma}$. Using the property of the Hukuhara-type difference, we have

$$
\begin{aligned}
d_{C\left(\mathbb{R}_{+}, X\right)}\left(T_{\gamma} x, T_{\gamma} y\right) & =\gamma^{-1} \sup _{t \in \mathbb{R}_{+}} d_{X}(x(t+\gamma) \stackrel{h}{-} x(t), y(t+\gamma) \stackrel{h}{-} y(t)) \\
& \leq \gamma^{-1} \sup _{t \in \mathbb{R}_{+}} d_{X}(x(t+\gamma), y(t+\gamma))+\gamma^{-1} \sup _{t \in \mathbb{R}_{+}} d_{X}(x(t), y(t)) \\
& \leq 2 \gamma^{-1} \sup _{t \in \mathbb{R}_{+}} d_{X}(x(t), y(t)) .
\end{aligned}
$$

Therefore,

$$
\left\|T_{\gamma}\right\| \leq 2 \gamma^{-1}
$$


Next, we estimate a deviation $d_{X}\left(D_{H} x(t), T_{\gamma} x(t)\right)$ on the class of functions $x \in K$ such that $D_{H} x \in$ $B H^{\omega}\left(\mathbb{R}_{+}, X\right)$. We have

$$
\begin{aligned}
d_{X}\left(D_{H} x(t), T_{\gamma} x(t)\right) & =d_{X}\left(D_{H} x(t), \gamma^{-1}\left(x(t+\gamma)-\frac{h}{-} x(t)\right)\right) \\
& =d_{X}\left(\gamma^{-1} \int_{t}^{t+\gamma} D_{H} x(t) d u, \gamma^{-1} \int_{t}^{t+\gamma} D_{H} x(u) d u\right) \\
& \leq \gamma^{-1} \int_{t}^{t+\gamma} d_{X}\left(D_{H} x(t) d u, D_{H} x(u)\right) d u \\
& \leq \gamma^{-1}\left\|D_{H} x\right\|_{H^{\omega}\left(\mathbb{R}_{+}, X\right)} \int_{t}^{t+\gamma} \omega(|t-u|) d u \\
& =\gamma^{-1}\left\|D_{H} x\right\|_{H^{\omega}\left(\mathbb{R}_{+}, X\right)} \int_{0}^{\gamma} \omega(u) d u .
\end{aligned}
$$

Thus,

$$
U\left(T_{\gamma}\right)=\sup _{t \in \mathbb{R}_{+}} d_{X}\left(D_{H} x(t), T_{\gamma} x(t)\right) \leq \gamma^{-1}\left\|D_{H} x\right\|_{H^{\omega}\left(\mathbb{R}_{+}, X\right)} \int_{0}^{\gamma} \omega(u) d u .
$$

Consider the function

$$
y(t)= \begin{cases}\omega(\gamma)-\omega(t), & t \leq \gamma \\ 0, & t \geq \gamma\end{cases}
$$

Choose $t_{0}$ such that

$$
\int_{0}^{t_{0}} y(u) d u=\int_{t_{0}}^{\gamma} y(u) d u
$$

Let

$$
z(t)=\left|\int_{t_{0}}^{t} y(u) d u\right|
$$

Choose an arbitrary element $x \in X$, that has inverse $x^{\prime}$, such that $\|x\|=1$. Then, the extremum function is the following

$$
x_{\gamma}(t)= \begin{cases}x z(t), & t \geq t_{0} \\ x^{\prime} z(t), & t \leq t_{0} .\end{cases}
$$

Let us find $\left\|x_{\gamma}(\cdot)\right\|_{C\left(\mathbb{R}_{+}, X\right)}, D_{H} x_{\gamma}(t),\left\|D_{H} x_{\gamma}(\cdot)\right\|_{C\left(\mathbb{R}_{+}, X\right)}$ and $\left\|D_{H} x_{\gamma}(\cdot)\right\|_{H^{\omega}}\left(\mathbb{R}_{+}, X\right)$. Because of $\left\|x_{\gamma}(t)\right\|=$ $z(t)$, we see that

$$
\left\|x_{\gamma}\right\|_{C\left(\mathbb{R}_{+}, X\right)}=\frac{1}{2} \int_{0}^{h}[\omega(h)-\omega(u)] d u
$$

We now evaluate $D_{H} x_{\gamma}(t)$. For $t>t_{0}$, we have

$$
\lim _{\tau \rightarrow 0+} \frac{z(t+\tau) x-z(t) x}{\tau}=\lim _{\tau \rightarrow 0+} \frac{z(t+\tau)-z(t)}{\tau} x=y(t) \cdot x .
$$

Similarly,

$$
\lim _{\tau \rightarrow 0+} \frac{z(t) x-z(t-\tau) x}{\tau}=\lim _{\tau \rightarrow 0+} \frac{z(t)-z(t-\tau)}{\tau} x=y(t) \cdot x
$$

Thus, for $t>t_{0}$

$$
D_{H} x_{\gamma}(t)=y(t) \cdot x .
$$


If $t<t_{0}$, then

$$
\begin{aligned}
& \lim _{\tau \rightarrow 0+} \frac{x^{\prime} z(t+\tau) \stackrel{h}{-} x^{\prime} z(t)}{\tau}=\lim _{\tau \rightarrow 0+} \frac{(\theta \stackrel{h}{-} x) z(t+\tau) \stackrel{h}{-}(\theta \stackrel{h}{-} x) z(t)}{\tau} \\
& =\lim _{\tau \rightarrow 0+} \frac{(\theta \stackrel{h}{-} x z(t+\tau)) \stackrel{h}{-}(\theta \stackrel{h}{-} x z(t))}{\tau} \\
& =\lim _{h \rightarrow 0+} \frac{x z(t+h) \stackrel{h}{-} x z(t)}{\tau} \\
& =\lim _{\tau \rightarrow 0+} \frac{x(z(t) \stackrel{h}{-} z(t+\tau))}{\tau} \\
& =\lim _{\tau \rightarrow 0+} \frac{-x(z(t+\tau) \stackrel{h}{-} z(t))}{\tau} \\
& =-x z^{\prime}(t) \\
& =y(t) \cdot x \text {. }
\end{aligned}
$$

Similarly, we show that

$$
\lim _{\tau \rightarrow 0+} \frac{x^{\prime} z(t) \stackrel{h}{-} x^{\prime} z(t-\tau)}{\tau}=y(t) \cdot x
$$

Terefore, in this case also

$$
D_{H} x_{\gamma}(t)=y(t) \cdot x \text {. }
$$

Let $t=t_{0}$. Considering first limit below similarly to the case $t>t_{0}$, and second limit below similarly to the case $t<t_{0}$, we see that

$$
D_{H} x_{\gamma}\left(t_{0}\right)=\lim _{\tau \rightarrow 0+} \frac{z\left(t_{0}+\tau\right) x-z\left(t_{0}\right) x}{\tau}=\lim _{h \rightarrow 0+} \frac{x^{\prime} z\left(t_{0}\right)-{ }^{h} x^{\prime} z\left(t_{0}-\tau\right)}{\tau}=y\left(t_{0}\right) \cdot x .
$$

Thus, for any $t \in \mathbb{R}_{+}$

$$
D_{H} x_{\gamma}(t)=y(t) \cdot x=[\omega(\gamma)-\omega(t)]_{+} \cdot x
$$

Consequently,

$$
\left\|D_{H} x(\cdot)\right\|_{C\left(\mathbb{R}_{+}, X\right)}=\omega(\gamma)
$$

Let us show that

$$
\left\|D_{H} x_{\gamma}\right\|_{H^{\omega}\left(\mathbb{R}_{+}, X\right)}:=\sup _{t^{\prime}<t^{\prime \prime}} \frac{d_{X}\left(D_{H} \bar{x}\left(t^{\prime}\right), D_{H} \bar{x}\left(t^{\prime}\right)\right)}{\omega\left(\left|t^{\prime \prime}-t^{\prime}\right|\right)}=1 .
$$

Note that

$$
\begin{aligned}
d_{X}\left(D_{H} x_{\gamma}\left(t^{\prime}\right), D_{H} x_{\gamma}\left(t^{\prime \prime}\right)\right) & =d_{X}\left(x y\left(t^{\prime}\right), x y\left(t^{\prime \prime}\right)\right) \\
& =\left|y\left(t^{\prime}\right)-y\left(t^{\prime \prime}\right)\right| d_{X}(x, \theta) \\
& =\left|\left[\omega(h)-\omega\left(t^{\prime}\right)\right]-\left[\omega(h)-\omega\left(t^{\prime \prime}\right)\right]\right| \\
& =\left|\omega\left(t^{\prime}\right)-\omega\left(t^{\prime \prime}\right)\right| \leq \omega\left(\left|t^{\prime \prime}-t^{\prime}\right|\right) .
\end{aligned}
$$

Hence,

$$
\frac{d_{X}\left(D_{H} x_{\gamma}\left(t^{\prime}\right), D_{H} x_{\gamma} x\left(t^{\prime \prime}\right)\right)}{\omega\left(\left|t^{\prime \prime}-t^{\prime}\right|\right)} \leq 1
$$


On the other hand

$$
\sup _{t^{\prime}, t^{\prime \prime}} \frac{d_{X}\left(D_{H} x_{\gamma}\left(t^{\prime}\right), D_{H} x_{\gamma}\left(t^{\prime \prime}\right)\right)}{\omega\left(\left|t^{\prime \prime}-t^{\prime}\right|\right)} \geq \frac{d_{X}(x \omega(\gamma), \theta)}{\omega(\gamma)}=\frac{\omega(\gamma)}{\omega(\gamma)} d_{X}(x, \theta)=1 .
$$

Accordingly, we have

$$
\left\|D_{H} x_{\gamma}(\cdot)\right\|_{H^{\omega}\left(\mathbb{R}_{+}, X\right)}=1 .
$$

Using inequality (3.4), we obtain, for any function $x \in W^{1} B H^{\omega}\left(\mathbb{R}_{+}\right)$,

$$
\left\|D_{H} x\right\|_{C\left(\mathbb{R}_{+}, X\right)} \leq U\left(T_{\gamma}\right)+\left\|T_{\gamma}\right\|\|x\|_{C\left(\mathbb{R}_{+}\right)} \leq \gamma^{-1} \int_{0}^{\gamma} \omega(u) d u+\frac{2}{\gamma}\|x(\cdot)\|_{C\left(\mathbb{R}_{+}, X\right)} .
$$

And for any function $x \in W^{1} H^{\omega}\left(\mathbb{R}_{+}\right)$,

$$
\left\|D_{H} x\right\|_{C\left(\mathbb{R}_{+}, X\right)} \leq \gamma^{-1} \int_{0}^{\gamma} \omega(u) d u\left\|D_{H} x_{\gamma}(\cdot)\right\|_{H^{\omega}\left(\mathbb{R}_{+}, X\right)}+\frac{2}{\gamma}\|x(\cdot)\|_{C\left(\mathbb{R}_{+}, X\right)} .
$$

Substituting $x_{\gamma}$ into the last inequality, we obtain

$$
\left\|D_{H} x_{\gamma}(\cdot)\right\|_{C\left(\mathbb{R}_{+}, X\right)}=U\left(T_{\gamma}\right)+\left\|T_{\gamma}\right\|\left\|x_{\gamma}\right\|_{C\left(\mathbb{R}_{+}, X\right)}=\gamma^{-1} \int_{0}^{\gamma} \omega(u) d u+\frac{2}{\gamma}\left\|x_{\gamma}(\cdot)\right\|_{C\left(\mathbb{R}_{+}, X\right)} .
$$

Taking into account Theorem 3.1 and Theorem 3.2 we have the following.

Theorem 5.1. For any function $x \in W^{1} B H^{\omega}\left(\mathbb{R}_{+}, X\right)$, the following inequality holds

$$
\left\|D_{H} x(\cdot)\right\|_{C\left(\mathbb{R}_{+}, X\right)}=\sup _{t}\left\|D_{H} x(t)\right\|_{X} \leq \gamma^{-1} \int_{0}^{\gamma} \omega(u) d u\left\|D_{H} x(\cdot)\right\|_{H^{\omega}\left(\mathbb{R}_{+}, X\right)}+\frac{2}{\gamma}\|x(\cdot)\|_{C\left(\mathbb{R}_{+}, X\right)} .
$$

This inequality is the best possible. It turns into equality for defined above function $x_{\gamma}(\cdot)$ :

$$
\left\|D_{H} x(\cdot)\right\|_{C\left(\mathbb{R}_{+}, X\right)}=\gamma^{-1} \int_{0}^{\gamma} \omega(u) d u+\frac{2}{\gamma}\left\|x_{\gamma}(\cdot)\right\|_{C\left(\mathbb{R}_{+}, X\right)} .
$$

Consequently, for any $\gamma>0$,

$$
E(2 / \gamma)=E_{\mathscr{L}}\left(D_{H}, 2 / \gamma, W^{1} B H^{\omega}\left(\mathbb{R}_{+}, X\right)\right)=\gamma^{-1} \int_{0}^{\gamma} \omega(u) d u
$$

If $\delta$ and $\gamma$ are related by

$$
\delta=\frac{1}{2} \int_{0}^{\gamma}[\omega(\gamma)-\omega(u)] d u
$$

then

$$
\Omega\left(\delta, D_{H}, W^{1} B H^{\omega}\left(\mathbb{R}_{+}, X\right)\right)=\mathscr{E}_{\delta}\left(\mathscr{L}, D_{H}, W^{1} B H^{\omega}\left(\mathbb{R}_{+}, X\right)\right)=\omega(\gamma)
$$

6. Stechion's and Related Problems in $C(\mathbb{R}, X)$.

For any $\gamma>0$, we define an operator $T_{\gamma}: C(\mathbb{R}, X) \rightarrow C(\mathbb{R}, X)$ :

$$
T_{\gamma} x(t)=(2 \gamma)^{-1}(x(t+\gamma) \stackrel{h}{-} x(t-\gamma)) .
$$

Similar to how it was done in the previous section, we obtain

$$
\left\|T_{\gamma}\right\| \leq \gamma^{-1}
$$

and for deviation $U\left(T_{\gamma}\right)$ of this operator from $D_{H}$ on the class $W^{1} B H^{\omega}(\mathbb{R}, X)$, we have

$$
U\left(T_{\gamma}\right)=\sup _{t \in \mathbb{R}_{+}} d_{X}\left(D_{H} x(t), T_{\gamma} x(t)\right) \leq \gamma^{-1} \int_{0}^{\gamma} \omega(|u|) d u .
$$


Consider the function

$$
y(t)= \begin{cases}\omega(\gamma)-\omega(|t|), & |t| \leq \gamma \\ 0, & |t| \geq \gamma\end{cases}
$$

Let

$$
z(t)=\left|\int_{0}^{t} y(u) d u\right|
$$

We choose an arbitrary element $x \in X$, that has inverse $x^{\prime}$, such that $\|x\|=1$. Then the extremum function is the following

$$
x_{\gamma}(t)= \begin{cases}x z(t), & t \geq 0, \\ x^{\prime} z(t), & t \leq 0 .\end{cases}
$$

Similarly to how it was done in the previous section, we find

$$
\begin{gathered}
\left\|x_{\gamma}\right\|_{C(\mathbb{R}, X)}=\int_{0}^{\gamma}[\omega(\gamma)-\omega(u)] d u, \\
\left\|D_{H} x_{\gamma}(\cdot)\right\|_{C(\mathbb{R}, X)}=\omega(\gamma),
\end{gathered}
$$

and

$$
\left\|D_{H} x_{\gamma}(\cdot)\right\|_{H^{\omega}(\mathbb{R}, X)}=1 .
$$

Using obtained estimations and equalities, as well as, Theorems 3.1 and 3.2, we can prove the following result.

Theorem 6.1. For any function $x \in W^{1} B H^{\omega}(\mathbb{R}, X)$, the following inequality holds

$$
\left\|D_{H} x(\cdot)\right\|_{C(\mathbb{R}, X)}=\sup _{t}\left\|D_{H} x(t)\right\|_{X} \leq \gamma^{-1} \int_{0}^{\gamma} \omega(u) d u\left\|D_{H} x(\cdot)\right\|_{H^{\omega}(\mathbb{R}, X)}+\gamma^{-1}\|x(\cdot)\|_{C(\mathbb{R}, X)} .
$$

This inequality is the best possible. It turns into equality for defined above function $x_{\gamma}(\cdot)$ :

$$
\left\|D_{H} x(\cdot)\right\|_{C(\mathbb{R}, X)}=\gamma^{-1} \int_{0}^{\gamma} \omega(u) d u+\gamma^{-1}\left\|x_{\gamma}(\cdot)\right\|_{C(\mathbb{R}, X)} .
$$

Hence, for any $\gamma>0$,

$$
E(1 / \gamma)=E\left(D_{H}, 1 / \gamma, W^{1} B H^{\omega}(\mathbb{R}, X)\right)=\gamma^{-1} \int_{0}^{\gamma} \omega(u) d u
$$

If $\delta$ and $\gamma$ are related in the following way

$$
\delta=\int_{0}^{\gamma}[\omega(\gamma)-\omega(u)] d u
$$

then

$$
\Omega\left(\delta, D_{H}, W^{1} B H^{\omega}(\mathbb{R}, X)\right)=\mathscr{E}_{\delta}\left(\mathscr{L}, D_{H}, W^{1} B H^{\omega}(\mathbb{R}, X)\right)=\omega(\gamma)
$$




\section{REFERENCES}

[1] V.V. Arestov, Approximation of unbounded operators by bounded operators and related extremal problems, Russian Math, Surveys, 51 (1996), 1093-1126.

[2] S.M. Aseev, Quasilinear operators and their application in the theory of multivalued mappings [in Russian], Proc. Steklov Institute Math. 167 (1985), 25-52.

[3] V.F. Babenko, N.P. Korneichuk, V.A. Kofanov, S.A. Pichugov, Inequalities for Derivatives and Their Applications [in Russian], Naukova Dumka, Kiev, 2003.

[4] V. Babenko, Numerical methods for solution of Volterra and Fredholm integral equations for functions with values in L-spaces, Appl. Math. Comput. 291 (2016), 354-372.

[5] V. Babenko, Calculus and nonlinear integral equations for functions with values in L-spaces, to appear in Analysis Mathematica.

[6] F.S. de Blasi, F. Iervolino, Equazioni differenziali con soluzioni a valore compatto convesso, Boll. Unione Mat.Ital. 4 (1969), 491-501.

[7] P. Diamond, P. Kloeden, Metric spaces of fuzzy sets, Fuzzy Sets Systems 35 (1990), 241-249.

[8] N. Dyn, E. Farkhi, A. Mokhov, Approximation of set-valued functions, Imperial College Press, London, 2014.

[9] M. Hukuhara, Integration des applicaitons mesurables dont la valeur est un compact convexe, Funkcialaj Ekvacioj, 10 (1967) 205-223.

[10] V. A. Plotnikov, A.V. Plotnikov, A.N. Vityuk, Differential equations with multivalued right-hand side [in Russian], Asymptotic Methods, AstroPrint, Odessa, 1999.

[11] A. Ramm, On numerical differentiation, Russian Math. (Izvestiya VUZ. Matematika), 78 (1968), 131-134.

[12] A.G. Ramm, A.B. Smirnova, On stable numerical differentiation, Math. Comput. 70 (2001), 1131-1153.

[13] S.B. Stechkin, Best approximation of linear operators, Math. Notes 1 (1967) 91-99.

[14] S.A. Vahrameev, Integration in $L$-spaces, Applied Mathematics and Mathematical Software of Computers [in Russian], MSU Publisher, pp. 45-47, 1980. 